American Journal of Food Technology 2 (7): 593-601, 2007

ISSN 1557-4571

(C) 2007 Academic Journals Inc.

\title{
Polyphenol Uses in Seafood Conservation
}

\author{
M.C. Gómez-Guillén and M.P. Montero \\ Instituto del Frío (CSIC), C/José Antonio Nováis, 1028040, Madrid
}

\begin{abstract}
Oxidative reactions are the main culprits in color alterations associated with seafood spoilage. Black spot formation or melanosis is a natural post-mortem oxidative mechanism, which cause crustaceans rejection by consumers because of their appearance. The use of phenolic compounds, such as 4-hexylresorcinol, appears to be a good alternative to the conventional sulphites, allowing longer conservation periods. Lipidic oxidation is also one of the main restrictive factors in the preservation of fish products. The application of natural plant extracts to prevent oxidative rancidity has been studied in a number of fish products. However, polyphenol-protein interactions may have a masking effect on the free radical scavenging activity of polyphenols and may also have a detrimental effect on the in vivo bioavailability of both phenolics and proteins. Polyphenolic plant extracts may be included into fish gelatin-based films in order to obtain an edible film with antioxidant properties. These films applied on the surface of raw or mild processed salted or smoked fish may increase the shelf life of fish products by reduction the lipid oxidation during the chilled storage.
\end{abstract}

Key words: Seafood, polyphenols, antioxidant properties, antimicrobial properties, melanosis, natural extracts

\section{INTRODUCTION}

Fish is a highly perishable food whose loss of freshness takes place much more quickly than in the case of mammal and avian meat. Fish and crustaceans are poikilothermic, lacking corporal thermoregulation and whose metabolism is adapted, therefore, to the low temperatures of sea water. This is an important factor in the development of the post-mortem biochemical processes and requires the employment of lower temperatures than for other foods in order to retard to the maximum fish spoilage. The loss of fish freshness is associated with sensorial alterations in the skin colour and gills, the softening of muscles due to proteolytic processes and development of unpleasant smells and flavours as a result of the accumulation of non-protein nitrogenous substances, as well as from compounds coming from the enzymatic degradation of proteins and lipids. Additionally, the high humidity content of fish muscle favours the growth of microorganisms and accelerates the biochemical reactions of degradation.

Oxidative reactions are the main culprits in the alteration of colour associated with the loss of seafood freshness, such as the loss of shine and colour intensity of the skin, the browning of the gills and dark muscle and the blackspot formation or melanosis in crustaceans and cephalopods. Lipidic oxidation is also one of the main restrictive factors in the preservation of fish products. Lipid content, fundamentally phospholipids and triglycerides, can be very high, varying from one species to another. So, in function of muscular fat content, fish can be classified as lean (0-2\%), semi-fat (2-6\%) and fattyacid (6-25\%). Furthermore, marine lipids are characterized by a high content of polyunsaturated fatty acids (PUFA), in particular eicosapentaenoic acid (EPA) and docosahexaenoic acid (DHA)

Corresponding Author: M.C. Gómez-Guillén, Instituto del Frío (CSIC), C/José Antonio Nováis, 1028040, Madrid Tel: +34 915492300 Fax: +34 915493627 
(Ackman, 1989). Degradation of PUFA by auto-oxidation during storage and processing is the main reason why rancid off-flavours develop due to the formation of volatile compounds. Moreover, the normally high concentration of metals acting as catalysts in seafood makes fish more prone to oxidation than meat (Ramanathan and Das, 1992).

To stabilize the high amount of PUFA, fatty fish contain an endogenous antioxidant system in vivo, made up of enzymes capable of eliminating reactive oxygen species and by compounds that act as free radical scavengers. Among the main antioxidants in fish muscle, those that figure prominently are $\alpha$-tocopherol, ubiquinone and carotenoids, all lipophilic in nature and glutathione and ascorbate, both hydrophilic antioxidants. These endogenous antioxidants, however, are consumed sequentially after the death of the fish and in this sense, the depletion of $\alpha$-tocopherol has shown a close correlation with lipid oxidation in minced muscle (Pazos et al., 2005b).

\section{Seafood Processing and Preservation}

Usual procedures for seafood preservation, such as refrigeration and freezing, are not effective in preventing lipidic oxidation, as rancid off-flavours can develop within several days or weeks, depending on the case. Also, certain technological processes involving thermal treatment (canning, cooking, microwave-treated products, etc.) produce lipid damage by PUFA oxidation, aided by the thermal disruption of the muscle membrane system, which exposes lipids to oxygen and catalysts such as iron (Sato and Hegarty, 1971).

In the case of canned seafood, it is important to consider the filling medium that is usually oil or brine. The filling medium may produce different dilutions and heat transfer in fish muscle, as well as the partial extraction of some components (Sánchez-Muniz et al., 1992). Brine was reported to make fish lipids more prone to oxidation than oil, probably due to the accumulation of unsaturated fatty acids at the oil-water interface (Medina et al., 1998). The nature of the packing oil is another factor to keep in mind. Extra Virgin Olive Oil (EVOO) has shown itself to be the best for preventing lipidic oxidation, not only in the oil itself but in the fish muscle that it accompanies, by virtue of the high concentration of natural polyphenols present in its composition (Medina et al., 1998). Other types of refined olive oil, as well as soybean oil, were not shown to be effective due to the loss of phenolic compounds after technological treatment. The natural polyphenols from EVOO may act as free radical acceptors and metal chelators, thus reducing lipid oxidation. This antioxidant ability was attributed to the solubilization of hydrophilic phenols in the water-muscle interface. However, its uses in fish processing is limited due to its strong fruity flavour and also its high cost. On the other hand, canning at high temperatures (around $100^{\circ} \mathrm{C}$ ) hydrolyses the complex phenols thus producing simple compounds, causing antioxidant properties to decrease due to a loss in their hydrogen-donating ability (Medina et al., 1999). Other non-thermal treatments have received attention in the last few years and in fact, have already begun to be incorporated on an industrial level in some foodstuffs. Such is the case with the use of high-pressure treatments to prolong the shelf life of cooked ham, oysters and some vegetable products (Barbosa-Cánovas et al., 2005). It has already been known for some time that, although high pressures are effective at inactivating or reducing microbial load, they can favour some muscle lipid oxidation during subsequent storage. Some studies point to the release of certain metals as a possible cause, mainly that of iron contained in the myoglobin that act as catalysts for oxidative reactions (Cheah and Ledward, 1996).

\section{Plant Extracts in Seafood Preservation}

Deterioration arising from lipid oxidation may be reduced by the incorporation of antioxidants. Nowadays, consumers are becoming increasingly concerned about synthetic chemicals in foods and are interested in the use of natural compounds. There is a growing interest in identifying antioxidative properties in many natural sources of polyphenolic compounds for food preservation 
(Peschel et al., 2006). The antioxidant properties of spices and herbs are related to their phenolic content and therefore their antioxidant action is similar to that of synthetic phenolic antioxidants. It is well accepted that the radical scavenging capability of phenolic compounds is due to their hydrogen donating ability: the larger the number of hydroxyl groups, the greater the possible free radical scavenging ability (Chen and Ho, 1995). Some flavonoids are reported to exhibit a strong tendency to suffer polymerization that produces variations in their radical scavenging activity. When the degree of polymerization exceeds a critical value, the availability of hydroxyl groups decreases and the antioxidant capacity is reduced. Moreover, in systems containing different phenolic species, interactions among these polyphenols may promote a negative synergistic effect on antioxidant capacity (Pinelo et al., 2004). On the other hand, polyphenols may form soluble or insoluble complexes with proteins (Shahidi and Naczk, 2004). These polyphenol-protein interactions may have a masking effect on the free radical scavenging activity of polyphenols (Arts et al., 2002). Furthermore, these interactions may also have a detrimental effect on the in vivo bioavailability of both phenolics and proteins, which should be taken into account when considering their technological uses in seafood preservation or fortification. The utilisation of species and herbs in fish products may also be limited by sensory considerations (Pérez-Mateos et al., 2001). For example, some of the compounds in rosemary extract have a strong odour and bitter taste; consequently there is particular interest in using low-odour, low-flavour extracts and in limiting the quantity in each product (Duxbury, 1989). When considering the use of quercetin extract to improve antioxidant properties in seafood products, the strong yellow coloration which it imparts to the product should also be taken into account (Pérez-Mateos et al., 2005).

The application of plant extracts to prevent fish oxidative rancidity has been studied in a number of fish products. For example, different polyphenols such as quercetin (200 ppm), myricetin $(200 \mathrm{ppm})$, tannic acid (30 and $200 \mathrm{ppm})$ and ellagic acid (30 and $200 \mathrm{ppm})$ were found to be potent antioxidants in raw and cooked ground fish (Scomberomorus commersoni) stored at either -20 or $4{ }^{\circ} \mathrm{C}$ (Ramanathan and Das, 1992). In this study, $\alpha$-tocopherol (30 ppm) was shown to be ineffective in inhibiting lipid oxidation in raw fish, whereas L-ascorbic acid acted as a pro-oxidant in steam and microwave-cooked fish. Grape procyanidins contained in skins and seeds have been successfully applied to prevent oxidation in fish oil and in frozen minced Atlantic mackerel muscle (Pazos et al., 2005a). These flavanol oligomers were shown to have a protective effect on endogenous muscle $\alpha$-tocopherol by delaying its depletion during storage (Pazos et al., 2005b), as a result of their capacity for restraining peroxide formation and/or for regenerating $\alpha$-tocopherol. Frozen horse mackerel fillets treated by spraying or glazing with an aqueous phenolic solution from grapes were found to be free of rancid odours for more than 21 weeks at $-10^{\circ} \mathrm{C}$ (Pazos et al., 2006). These authors reported that a previous washing of the fillets improved the antioxidant effectiveness of the grape procyanidins. On the other hand, catechins contained in different tea extracts were reported to produce an excellent oxidative stability in ground mackerel muscle cooked at $75^{\circ} \mathrm{C}$ and stored at $42^{\circ} \mathrm{C}$ for seven days, as compared to other antioxidants such as alpha-tocopherol, butylated hydroxytoluene (BHT), butylated hydroxyanisole (BHA) or tert-butylhydroquinine (TBHQ) (He and Shahidi, 1997). Similarly, impregnation of blue sprat (Spratelloides gracilis) with different hot water tea extracts was also found to be effective in suppressing deterioration by lipid oxidation during storage at $5^{\circ} \mathrm{C}$ (Seto et al., 2005). The antioxidant activity was highest with Tungting oolong tea extract and was positively correlated with catechin content, especially with that of epigallocatechin gallate (EGCG), but not with total polyphenol content. EGCG from green tea was also revealed as a potent inhibitor of metalloproteinase activity in rainbow trout muscle during chilled storage, playing an important role in suppressing fish muscle softening arising from proteolytic degradation of collagen (Saito et al., 2002). Rosemary extract, containing a high amount of phenolic diterpenes such as carnosic acid and carnosol, has been reported to exhibit potent antioxidant activity in rainbow trout muscle during refrigerated and frozen storage 
(Akhtar et al., 1998), as well as in frozen horse mackerel and in Mediterranean hake (Vareltzis et al., 1997). A commercial rosemary liquid extract was applied to prolong the shelf-life of refrigerated gilt-head sea bream fillets packaged in a modified atmosphere (Giménez et al., 2004). Rosemary extract was more effective than ascorbic acid in delaying lipid oxidation under different lighting conditions, however, no differences were found in microbial counts as a result of the application of either rosemary or ascorbic acid during storage. Many natural compounds found in dietary plants have manifested antimicrobial activities with respect to L. monocitogenes (Hao et al., 1998). In this sense, oregano and cranberry aqueous extracts mixed in a ratio of $75 \%$ oregano and $25 \%$ cranberry proved effective in inhibiting the growth of $L$. monocytogenes in cod fillets stored at $4^{\circ} \mathrm{C}$. Their antimicrobial efficacy was further enhanced when combined with lactic acid (Lin et al., 2004). According to these authors, the partial hydrophobic nature of the phenolic constituents in the oregano/cranberry mixture facilitates accumulation and attachment to the bacterial cytoplasmic membrane, where inhibitory effects may eventually lead to cell death. Moreover, phenolic-containing extracts at high concentrations may decrease the $\mathrm{pH}$ owing to proton donation and cell membrane disruption.

\section{Antioxidant and Antimicrobial Properties in Smoked Fish Products}

Smoking is one of the oldest methods of preserving food, by means of lowering water activity levels and by the antimicrobial and antioxidant effects of the smoke components (Suñén, 1998), such as phenols and others like aldehydes, ketones, hydrocarbons and esters, which are deposited on the surface and later penetrate into the muscle (Guillén et al., 2006). The most common method of fish smoking today is cold smoking at temperatures of around $25-30^{\circ} \mathrm{C}$. These temperatures are not high enough to kill the micro-organisms, although the combined effect of $\mathrm{NaCl}$ and smoke components acts synergistically on the shelf life of smoked fish (Giménez and Dalgaard, 2004; Montero et al., 2006a). Some smoke extracts, depending on the type of smoke and phenol concentrations, also prove to be an additional barrier to L. monocytogenes and Aeromonas hydrophila growth in cold-smoked rainbow trout (Suñén et al., 2003). The presence of antioxidant phenolic compounds in smoke may be able to maintain low levels of lipid oxidation, however, in certain fish species subjected to mild processing conditions, oxidative instability may arise (Guillén et al., 2006). The addition of aqueous extracts from rosemary and oregano leaves to the brines used for prior fish salting was found to prevent lipid oxidation during chilled storage of cold-smoked sardines (unpublished data).

By applying a gelatin-based film containing a certain amount of either oregano or rosemary aqueous extracts to the surface of cold smoked sardines was also found to produce a noticeable reduction in lipid oxidation during chilled storage (Gómez-Estaca et al., 2006). The addition of rosemary extract $(2.5 \%)$ or oregano extract $(1.25 \%)$ to gelatin-based films considerably increased the antioxidant properties of the resulting film, as measured by the FRAP assay, causing only minor modifications in its mechanical properties (Fernández-Martín et al., 2006). Other polyphenolic plant extracts may be included in edible films in order to increase their antioxidant properties. This would be the case of films made from tuna-fish gelatin with the addition of two different murta (Ugni molinae Turcz) ecotype leaf extracts (Gómez-Guillén et al., 2006). The use of an extract with a higher polyphenol content was found to increase the antioxidant capacity of the film, but had a detrimental effect on its mechanical properties, because of a greater interaction between polyphenols and proteins.

\section{Antioxidant Properties in Restructured Fish Products}

In recent years there has been an increasing interest in the consumption of restructured minced seafood products, largely based on thermally-induced gelation of proteins. Oxidation of muscle components, especially lipids and proteins, may be considerably increased after this type of processing. In fact, protein oxidation may occur more rapidly than lipid oxidation in fish muscle, since 
the protein is in the aqueous phase where many radicals are formed (Srinivasan and Hultin, 1995). For the elaboration of gel-based restructured fish products, previous solubilization of the muscle proteins with $2-3 \%$ salt is normally required. Montero et al. (2005) reported a noticeable increase in the TBA index of salt ground muscle from Atlantic mackerel, which was efficiently prevented by the addition of either rosemary or quercetin extracts.

On the other hand, high-pressure treatment has been applied successfully as an alternative to thermal treatment for the production of fish gels at low or moderate temperatures (Montero and Gómez-Guillén, 2005). This could have special repercussions in the production of functional foodstuffs made from fish, with the aim of maintaining intact, as much as possible, the bioactive properties of the healthy compounds incorporated in the product. Nevertheless, it has been observed that pressure-induced gels are more prone to oxidation than cooked gels. The incorporation of an extract from rosemary was found to be effective in controlling lipid oxidation in high pressure-induced gels from Atlantic mackerel flesh (Pérez-Mateos et al., 2002), causing only slight alterations in the rheological characteristics of the final product. The antioxidant activity of rosemary and quercetin extracts was compared in minced fish after subsequent thermal and high-pressure treatments to form gels (Montero et al., 2005). In this study, the rosemary extract proved more effective at protecting heat-induced and high-pressure gels from lipid oxidation, whereas quercetin seemed to be most effective against oxidation of proteins, especially in gels induced by conventional heat treatment. The antioxidant properties of quercetin incorporated as a functional ingredient in omega- 3 enriched southern blue whiting (Micromesistius australis) gels was evidenced by the ferric-reducing/antioxidant power (FRAP) method (Pérez-Mateos et al., 2005). When these gels were fed to rats, total serum antioxidant capacity was increased, without significant alteration of the serum lipid profile. These results suggest that fortification of seafood products with natural antioxidants may contribute to the overall protective activity of such antioxidants in the body.

\section{Melanosis in Crustaceans}

Crustaceans experience a more or less intense characteristic colour alteration. Black spots or melanosis in crustaceans is a natural postmortem mechanism that involves the action of an enzymatic complex, namely polyphenoloxidase (PPO), which in the presence of oxygen, oxidizes peptide and aminoacid phenols converting them into quinones. Polymerization of the colourless quinones gives rise to black high-molecular-weight pigments (McEvily et al., 1991). The pigments themselves are not dangerous to human health, but cause the crustaceans to be rejected by consumers because of their appearance. Melanosis development depends greatly on interspecies variations, cyclical changes in physiological susceptibility, the melanosis inhibitor type and concentration and the method of application employed (Montero et al., 2004). Sulphite-based formulations are currently used to avoid or at least delay melanosis, however, high concentrations of additives are sometimes required to prevent melanosis effectively (Gómez-Guillén et al., 2005). For this reason, m-diphenol compounds, such as 4-hexylresorcinol (4-HR), seem to be good alternatives. Their phenolic nature causes enzyme inhibition by competing with the substrates naturally present in crustaceans. In the case of 4-HR, it interacts with PPO, as much in the formation of diphenols as in that of quinones, making the enzyme incapable of catalysing the enzymatic reactions (Lambrecht, 1995). Other studies exist in which the inhibiting capacity of alternative phenolic compounds in crustaceans have been evaluated. Sodium benzoate and kojic acid were effective when used separately and together in the partial inhibition of melanosis in Penaeus japonicus prawns (Montero et al., 2001). However, various compounds such as kojic acid, benzoic acid, caffeic acid, fitico acid and cumic acid were hardly effective as melanosis inhibitors in deepwater pink shrimp (Parapenaeus longirostris) (Montero et al., 2003). The way sodium benzoate acts is similar to that of other phenolic derivatives, such as cumic acid and kojico acid. These compounds act predominantly as copper chelating agents by way of unions with hydroxyl 
groups at the active center of the enzyme or to a lesser degree through the formation of Schiff bases by means of aldehyde groups. Moreover, kojic acid inhibits the formation of coloured pigments by capturing the oxygen from the medium when o-dihydroxyphenols and trihydroxyphenols are oxidised by PPO (Chen et al., 1991).

As far as 4-hexylresorcinol is concerned, its use permits longer preservation periods than the conventional sulphites, showing lower microbial growth and higher sensory scores. Thus, applying $0.25 \% 4$-HR proved to be more efficient at inhibiting black spot formation than applying a $4 \%$ concentration of a commercial sodium metabisulphite-based product. Additionally, 4-HR was a more effective inhibitor of enterobacteria and lactic acid bacteria than sulphites, which, conversely were more effective against luminescent bacteria (Martínez-Alvarez et al., 2005a). Similarly, a formulation based on $4-H R$ at a $0.1 \%$ concentration, applied in vivo to cultured tiger prawns (Marsupenaeus japonicus), delayed the appearance of melanosis during the first week of chilled storage and caused a reduction in the growth of total viable bacteria, H2S-producing microorganisms, lactic acid bacteria, enterobacteria and pseudomonads (Martínez-Alvarez et al., 2005b). The minimum dose of 4-HR for preventing melanosis may vary from one species to another. In deepwater pink shrimp, which is highly susceptible to melanosis, the most effective amount to give at least one week without melanosis was found to be $0.5 \%$, with a residual level in muscle ranging from $\approx 80$ to $90 \mathrm{mg} \mathrm{kg}^{-1}$ on day 2 of storage to $>200 \mathrm{mg} \mathrm{kg}^{-1}$ after 7 days. Residues of around $2 \mathrm{mg} \mathrm{kg}^{-1}$, which have recently been permitted by the EU, were shown to be effective in preventing melanosis in pink shrimp for only 2 days from a commercial point of view (Montero et al., 2006b).

\section{ACKNOWLEDGMENTS}

This study has been sponsored by the Spanish Ministry of Education and Science under project AGL2005-02380/ALI.

\section{REFERENCES}

Ackman, R.G., 1989. Marine Biogenic Lipids, Fats and Oils. CRC Press, Boca Raton, FL.

Akhtar, P., J.I. Gray, E.A. Gomaa and A.M. Booren, 1998. Effect of dietary components and surface application of oleoresin rosemary on lipid stability of rainbow trout (Oncorhynchus mykiss) muscle during refrigerated and frozen storage. J. Food Lipids, 5: 43-58.

Arts, M.J.T.J., G.R.M.M. Haenen, L.C. Wilms, S.A.J.N. Beestra, C.G.M. Heijnen, H.P. Voss and A. Bast, 2002. Interactions between flavonoids and proteins: Effect on the total antioxidant capacity. J. Agric. Food Chem., 50: 1184-1187.

Barbosa-Canovas, G.V., M.S. Tapia and M.P. Cano, 2005. Novel Food Processing Technologies. Marcel Dekker/CRC Press, Boca Raton, Florida.

Chen, J.S., C.I. Wei and M.R. Marshall, 1991. Inhibitory mechanism of kojic acid on polyphenol oxidase. J. Agric. Food Chem., 39: 1897-1901.

Chen, C.W. and C.T. Ho, 1995. Antioxidant properties of polyphenols extracted from green and black tea. J. Food Lipids, 1: 35-46.

Cheah, P.B. and D.A. Ledward, 1996. High pressure effects on lipid oxidation in minced pork. Meat Sci., 43: 123-134.

Duxbury, D.D., 1989. Decolorized, deflavorized rosemary extract antioxidant properties retard oxidative rancidity. Food Processing, 50: 62-64.

Fernández-Martín, F., J. Gómez-Estaca, M.C. Gómez-Guillén and P. Montero, 2006. Some physical properties of gelatin based edible films with functional applications. Calorimetry and termal analysis conference (Calcat'06) Tools in Science, Industry and Environmental studies. Santiago de Compostela (Spain), 9-12 July. 
Giménez, B. and P. Dalgaard, 2004. Modelling and predicting the simultaneous growth of Listeria monocytogenes and spoilage micro-organisms in cold-smoked salmon. J. Applied Microbiol., 96: 96-109.

Giménez, B., P. Roncalés and J.A. Beltrán, 2004. The effects of natural antioxidants and lighting conditions on the quality characteristics of gilt-sea bream fillets (Sparus aurata) packaged in a modified atmosphere. J. Sci. Food Agric., 84: 1053-1060.

Gómez-Estaca, J., M.C. Gómez-Guillén and P. Montero, 2006. Use of high pressure and protective edible films for preserving cold-smoked fish. XI Congreso Anual en Ciencia y Tecnología de los Alimentos (Annual Congress in Food Science and Technology), Madrid, 29 march-1 April. Oral communication.

Gómez-Guillén, M.C., O. Martínez-Alvarez, A. Llamas and P. Montero, 2005. Melanosis inhibition and $\mathrm{SO}_{2}$ residual levels in shrimps (Parapenaeus longirostris) after different sulfite-based treatments. J. Sci. Food Agric., 85:1143-1148.

Gómez-Guillén, M.C., M. Ihl, V. Bifani, A. Silva and P. Montero, 2006. Edible films made from tuna-fish gelatin with antioxidant extracts of two different murta ecotypes leaves (Ugni molinae Turcz). Food Hydrocolloids (In Press).

Guillén, M.D., M.C. Errecalde, J. Salmerón and C. Casas, 2006. Headspace volatile components of smoked swordfish (Xiphias gladius) and cod (Gadus morhua). Food Chem., 94: 151-156.

Hao, Y.Y., R.E. Brackett and M.P. Doyle, 1998. Inhibition of Listeria monocytogenes and Aeromonas hydrophila by plant extracts in refrigerated cooked beef. J. Food Prot., 61: 307-312.

He, Y. and F. Shahidi, 1997. Antioxidant activity of green tea and its catechins in a fish meat model system. J. Agric. Food Chem., 45: 4262-4266.

Lambrecht, H.S., 1995. Sulfite Substitutes for the Prevention of Enzymatic Browning in Foods. In: Enzymatic Browning and its Prevention. Lee, C.Y. and J.R. Whitaker (Eds.), American Chemical Society, Washington DC., pp: 313-323.

Lin, Y.T., R.G. Labbe and K. Shetty, 2004. Inhibition of Listeria monocytogenes in fish and meat systems by use of oregano and cranberry phytochemical synergies. Applied Environ. Microbiol., 70: $5672-5678$.

Martínez-Alvarez, O., M.C. Gómez-Guillén and P. Montero, 2005a. Role of sulfites and 4-hexylresorcinol on microbial growth and melanosis prevention in shrimps using controlled atmosphere. J. Food Prot., 68: 103-110.

Martínez-Alvarez, O., M.E. López-Caballero, P. Montero and M.C. Gómez-Guillén, 2005 b. A 4-hexyl-resorcinol based formulation to prevent melanosis and microbial growth in chilled tiger prawns from aquaculture. J. Food Sci., 70: 415-422.

McEvily, A., I. Radha and S. Otwell, 1991. Sulfite alternative prevents shrimps melanosis. Food Technol., 9: 80-86.

Medina, I., R. Sacchi, L. Biondi, S.P. Aubourg and L. Paolillo, 1998. Effect of packing media on the oxidation of canned tuna lipids. Antioxidant effectiveness of extra virgin olive oil. J. Agric. Food Chem., 46: 1150-1157.

Medina, I., M.T. Satué-Gracia, J.B. German and E.N. Frankel, 1999. Comparison of natural polyphenol antioxidants from extra virgin olive oil with synthetic antioxidants in tuna lipids during thermal oxidation. J. Agric. Food Chem., 47: 4873-4879.

Montero, P., A. Avalos and M. Pérez-Mateos, 2001. Characterization of polyphenoloxidase of prawns (Penaeus japonicus). Alternatives to inhibition: Additives and high-pressure treatment. Food Chem., 75: 317-324.

Montero, P., M.C. Gómez-Guillén, P. Zamorano and O. Martínez-Álvarez, 2003. Estudios de los agentes conservantes e inhibidores de la melanosis en crustáceos. Ed. Junta de Andalucia. Consejería de Agricultura y Pesca (Spain). 
Montero, P., O. Martínez-Alvarez and M.C. Gómez-Guillén, 2004. Effectiveness of on-board application of 4-hexylresorcinol in inhibiting melanosis in shrimp (Parapenaeus longirostris). J. Food Sci., 69: 643-647.

Montero, P. and M.C. Gómez-Guillén, 2005. Application of High Pressure to Myosistems. In: Novel Food Processing Technologies. Marcel Dekker, CRC Press, Boca Ratón, FL.

Montero, P., B. Giménez, M. Pérez-Mateos and M.C. Gómez-Guillén, 2005. Oxidation stability of muscle with quercetin and rosemary during thermal and high-pressure gelation. Food Chem., 93: 17-23.

Montero, P., J. Gómez-Estaca and M.C. Gómez-Guillén, 2006a. Influence of salt, smoke and high pressure on Listeria monocytogenes and spoilage microflora in cold-smoked dolphinfish. J. Food Prot., (In Press).

Montero, P., O. Martínez-Álvarez, J.P. Zamorano, R. Alique and M.C. Gómez-Guillén, $2006 \mathrm{~b}$. Melanosis inhibition and 4-hexylresorcinol residual levels in deepwater pink shrimp (Parapenaeus longirostris) following various treatments. Eur. Food. Res. Technol., 223: 16-21.

Pazos, M., J.M. Gallardo, J.L. Torres and I. Medina, 2005a. Activity of grape polyphenols as inhibitors of the oxidation of fish lipids and frozen fish muscle. Food Chem., 92: 547-557.

Pazos, M., M.J. González, J.M. Gallardo, J.L. Torres and I. Medina, 2005b. Preservation of the endogenous antioxidant system of fish muscle by grape polyphenols during frozen storage. Eur. Food. Res. Technol., 220: 514-519.

Pazos, M., A. Alonso, J. Fernández-Bolaños, J.L. Torres and I. Medina, 2006. Physicochemical properties of natural phenolics from grapes and olive oil byproducts and their antioxidant activity in frozen horse mackerel fillets. J. Agric. Food Chem., 54: 366-373.

Pérez-Mateos, M., L. Boyd, J.C. Allen and T.C. Lanier, 2001. Omega-3 fortification of surimi seafood. 46th Atlantic Fisheries Technology Conference (AFT) 26-29 August, Rimouski, Québec, Canada.

Pérez-Mateos, M., M.C. Gómez-Guillén, J.L. Hurtado, M. Solas and P. Montero, 2002. The effect of rosemary extract and omega- 3 unsaturated fatty acids on the properties of gels made from the flesh of mackerel (Scomber scombrus) by high pressure and heat treatments. Food Chem., 79: 1-8.

Pérez-Mateos, M., L. Bravo, L. Goya, M.C. Gómez-Guillén and P. Montero, 2005. Quercetin properties as a functional ingredient in omega-3 enriched fish gels fed to rats. J. Sci. Food Agric., 85: 1651-1659.

Peschel, W., F. Sanchez-Rabaneda, W. Diekmann, A. Plescher, I. Gartzia, D. Jiménez, R. Lamuela-Raventos, S. Buxaderas and C. Codina, 2006. An industrial approach in the search of natural antioxidants from vegetable and fruit wastes. Food Chem., 97: 137-150.

Pinelo, M., L. Manzocco, M.J. Núñez and M.C. Nicoli, 2004. Interaction among phenols in food fortification: Negative synergism on antioxidant capacity. J. Agric. Food Chem., 52: 1177-1180.

Ramanathan, L. and N.P. Das, 1992. Studies on the control of lipid oxidation in ground fish by some polyphenolic natural products. J. Agric. Food Chem., 40: 17-21.

Saito, M., K. Saito, N. Kunisaki and S. Kimura, 2002. Green tea polyphenols inhibit metalloproteinase activities in the skin, muscle and blood of rainbow trout. J. Agric. Food Chem., 50: 7169-7174.

Sánchez-Muniz, F., J. Viejo and R. Medina, 1992. Deep-frying of sardines in different culinary fats. Changes in the fatty acid composition of sardines and frying fats. J. Aric. Food Chem., 40: $2252-2256$.

Sato, K. and G.R. Hegarty, 1971. Warmed-over flavour in cooked meat. J. Food Sci., 36: 1098.

Seto, Y., C.C. Lin, Y. Endo and K. Fujimoto, 2005. Retardation of lipid oxidation in blue sprat by hot water tea extracts. J. Sci. Food Agric., 85: 1119-1124. 
Shahidi, F. and M. Naczk, 2004. Phenolics in Food and Nutraceuticals. CRC Press. Boca Raton, FL. Srinivasan, S. and H. Hultin, 1995. Hydroxil radical modification of fish muscle protein. J. Food Biochem., 18: 405-425.

Suñén, E., 1998. Minimum inhibitory concentration of smoke wood extracts against spoilage and pathogenic micro-organisms associated with foods. Lett. Applied Microbiol., 27: 45-48.

Suñén, E., C. Aristimuño and B. Fernández-Galian, 2003. Activity of smoke Word condensates against Aeromonas hydrophila and Listeria monocytogenes in vacuum-packaged, cold-smoked rainbow trout stored at $4^{\circ} \mathrm{C}$. Food Res. Int., 36: 111-116.

Vareltzis, K., D. Koufidis, E. Gavriilidou, E. Papavergou and S. Vasiliadou, 1997. Effectiveness of a natural Rosemary (Rosmarinus officinalis) extract on the stability of filleted and minced fish during frozen storage. Z. Lebensm. Unters. Forsch., 205: 93-96. 